\title{
DESENVOLVIMENTO DE EQUIPAMENTO MOTORIZADO PARA APLICAÇÃO LÍQUIDA DE PRODUTOS FITOSSANITÁRIOS NA CULTURA DO CAFÉ
}

\author{
TOMÁS K. MATUO ${ }^{1}$, CARLOS G. RAETANO ${ }^{2}$, TOMOMASSA MATUO ${ }^{3}$, \\ NILZA M. MARTINELLI ${ }^{4}$, GILSON J. LEITE ${ }^{5}$
}

\begin{abstract}
RESUMO: O trabalho teve como objetivo desenvolver um protótipo aplicador de líquidos na superfície do solo para cafezais localizados em regiões de topografia acidentada ou cultivados em sistema de plantio adensado. O protótipo do aplicador construído é um trator de rabiças acionado por um motor de dois tempos a gasolina, de $2,61 \mathrm{~kW}(3,5 \mathrm{cv})$, com duas rodas motrizes com bitola de $0,60 \mathrm{~m}$ e uma terceira roda direcional, equipado com sistema de pulverização dotado de depósito de 40 L, bomba centrífuga, filtros de linha, regulador de pressão, manômetro, mangueiras, válvulas e suporte de bicos. Para avaliar a eficiência do protótipo, foram instalados dois experimentos de campo em São Sebastião do Paraíso - MG (2003 e 2004), onde foram aplicados inseticidas sistêmicos para o controle da cigarra. Os métodos de aplicação avaliados foram: aplicação em faixa, filete, "drench" contínuo e "drench" intermitente. O protótipo aplicador de líquidos à superfície do solo atendeu aos objetivos propostos, visto que conseguiu realizar o controle químico de cigarras com destaque para a aplicação em "drench" contínuo. O protótipo, se equipado para aplicar simultaneamente nos dois lados da planta, apresentará capacidade de campo operacional semelhante à do equipamento tratorizado.
\end{abstract}

PALAVRAS-CHAVE: tecnologia de aplicação, equipamento aplicador, Quesada gigas.

\section{DEVELOPMENT OF A WALK BEHIND TRACTOR FOR SPRAYING COFFEE TREES}

ABSTRACT: The present research aimed to develop a motorized sprayer for coffee plantations located in sloping areas or planted in a high density planting system. The equipment developed is a walk behind tractor powered by a $2.61 \mathrm{~kW}(3.5 \mathrm{cv})$ two-stroke engine, clearance of $0.60 \mathrm{~m}$ between two driving wheels, having a third directional wheel, equipped with spray system composed by a 40 L hopper, centrifugal pump, line filters, pressure regulator and gauge, hoses, valves and nozzles. Evaluations of the prototype were made in two field tests in São Sebastião do Paraíso - MG, Brazil (2003 and 2004), applying systemic insecticides to control the coffee cicadas. The application technologies evaluated were: flat fan nozzle band application, solid stream nozzle application, continuous drench application and intermittent drench application. The walk behind tractor for spraying ground surface of coffee plantations reached the aim because it provided good chemical control of coffee cicadas. The continuous drench application was the most interesting method due to its simplicity. The prototype will present the overall work rate similar to the tractor powered equipment if equipped to spray both sides of the plant rows.

KEYWORDS: application technology, pesticide applicator, Quesada gigas.

\footnotetext{
${ }^{1}$ Eng $^{\mathrm{o}}$ Agrônomo, Doutor em Proteção de Plantas, FCA/UNESP, Jaboticabal - SP, tomaskmatuo@hotmail.com, bolsista do CNPq.

${ }^{2}$ Prof. Doutor, Departamento de Produção Vegetal, Defesa Fitossanitária, FCA/UNESP, Botucatu - SP.

${ }^{3}$ Prof. Titular, Departamento de Fitossanidade, FCAV/UNESP, Jaboticabal - SP.

${ }^{4}$ Profa. Doutora, Departamento de Fitossanidade, FCAV/UNESP - Jaboticabal -SP.

${ }^{5}$ Técnico Agrícola, Departamento de Fitossanidade, FCAV/UNESP - Jaboticabal - SP.

Recebido pelo Conselho Editorial em: 25-5-2007
}

Aprovado pelo Conselho Editorial em: 26-7-2008 


\section{INTRODUÇÃO}

Uma parte significativa dos produtos fitossanitários utilizada na cafeicultura é de formulação granulada, que é aplicada ao solo por meio de diferentes tipos de equipamentos. $\mathrm{O}$ tipo de equipamento a ser utilizado está diretamente relacionado com as características da área a ser tratada, onde a topografia, o espaçamento e a forma de plantio são fatores determinantes na seleção do melhor método de aplicação (MATUO, 2001).

Em áreas com relevos mais planos e que apresentam espaçamentos favoráveis ao uso de máquinas, são utilizados os equipamentos tratorizados. Porém, em áreas onde a topografia é mais acidentada e os espaçamentos menores, são utilizados equipamentos aplicadores manuais e, em menor escala, equipamentos de tração animal. Entretanto, as formulações do tipo granulado são constituídas de ingredientes ativos muito tóxicos, que acarretam alguns problemas quanto a sua utilização, especialmente em relação à exposição do aplicador (MACHADO NETO et al., 1996).

Recentemente, surgiram no mercado alguns inseticidas e também misturas desses com fungicidas para a aplicação líquida no solo, em substituição aos produtos formulados em grânulos. Essas novas formulações para aplicação líquida caracterizam-se pela baixa toxicidade. Entretanto, a forma de distribuição desses produtos ainda não está bem estabelecida.

No presente trabalho, foi desenvolvido um protótipo motorizado para a aplicação de líquido, derivado do aplicador de granulados descrito por MATUO (2001), apto a aplicar o produto sob diferentes formas sobre o solo do cafezal, sendo seu desempenho avaliado no controle da cigarra do cafeeiro.

\section{MATERIAL E MÉTODOS}

O aplicador foi montado sobre um microtrator de rabiças acionado por um motor da marca Branco, a gasolina, de dois tempos, com $2,61 \mathrm{~kW}$, com embreagem centrífuga e com duas rodas (pneus 4,10 x 3,50-8). Existe, também, uma terceira roda, que tem por função dar apoio e direcionar a máquina. A velocidade de deslocamento foi ajustada para aproximadamente $1,0 \mathrm{~m} \mathrm{~s}^{-1}$, que é a velocidade de caminhamento usual em condições de campo. Sobre o chassi apoiado às rodas, foi montada a estrutura do aplicador de formulações líquidas. O depósito do aplicador consiste num tanque de fibra de vidro de seção trapezoidal, com capacidade de $40 \mathrm{~L}$, e com o fundo afunilado. O sistema de pulverização, constituído por bomba, filtro, manômetro, regulador de pressão, mangueiras, registros e bicos, foi instalado no protótipo. A bomba utilizada foi da marca Shurflo, com capacidade de bombeamento de $0,167 \mathrm{~L} \mathrm{~s}^{-1}$.

O conjunto tem rabiças pelas quais o operador conduz a máquina e tem a sua mão o comando do acelerador, do afogador, do botão que desliga o motor e todos os comandos do sistema de pulverização: o acionamento da bomba e também o comando de abertura e fechamento da pulverização.

Com essas características, a máquina está preparada para operar em médias propriedades, em terrenos declivosos, em cafezais superadensados e em situações difíceis de operar com a máquina tratorizada.

Para avaliar a eficiência do protótipo no controle de cigarras, foram conduzidos dois ensaios de campo, com várias formas de aplicação líquida de inseticidas, comparando-as com as aplicações de formulações granuladas consideradas padrões de controle.

O primeiro ensaio de campo foi instalado em 2003, na Fazenda Santana da Bela Vista, município de São Sebastião do Paraíso - MG, em cafezal Mundo Novo, com 28 anos de idade, plantado em espaçamento de 3,70 x 2,50 m, e empregadas as seguintes formas de aplicação:

1) Aplicação em filete: o pulverizador foi equipado com um bico TX 10 (Spraying Systems), do qual se removeu o núcleo, de forma que ficasse somente o orifício circular para a produção de um filete líquido, posicionado em um suporte horizontal no lado direito da máquina, a $0,40 \mathrm{~m}$ ao 
lado, e $0,10 \mathrm{~m}$ atrás da roda direita e a $0,40 \mathrm{~m}$ de altura do solo. A pressão de trabalho foi de $200 \mathrm{kPa}$, mantendo-se a velocidade de $0,97 \mathrm{~m} \mathrm{~s}^{-1}$. O pulverizador foi regulado para aplicar $0,000084 \mathrm{~kg} \mathrm{~m}^{-2}$ de imidacloprida $\left(0,000120 \mathrm{~kg} \mathrm{~m}^{-2}\right.$ de Premier GrDA), diluído a $0,70 \%$, sendo o bico posicionado na projeção da copa.

2) Aplicação em faixa: o pulverizador foi equipado com um bico de jato plano (leque) 8004VB, posicionado no mesmo suporte descrito anteriormente, operando a $400 \mathrm{kPa}$ e velocidade de $0,97 \mathrm{~m} \mathrm{~s}^{-1}$. O equipamento foi regulado para aplicar $0,000084 \mathrm{~kg} \mathrm{~m}^{-2}$ de imidacloprida $\left(0,000120 \mathrm{~kg} \mathrm{~m}^{-2}\right.$ de Premier GrDA), diluído a $0,70 \%$. O bico foi posicionado para que a parte central da faixa de aplicação ficasse na projeção da copa.

3) Aplicação em forma de "drench" contínuo: outro suporte foi instalado na máquina, fixando o bico na posição horizontal, na ponta flexível do suporte. Nessa posição, foi colocado um bico "off-center" OC-04 (Spraying Systems), que é um bico de jato descentrado com maior projeção do jato em um dos lados, visando a atingir o tronco do cafeeiro. O pulverizador foi regulado para aplicar $0,000084 \mathrm{~kg} \mathrm{~m}^{-2}$ do mesmo produto químico, a $0,70 \%$, à velocidade de $0,97 \mathrm{~m} \mathrm{~s}^{-1}$. O bico foi deslocado sob a saia, de modo que o jato alcançasse o tronco das plantas.

4) Aplicação em forma de "drench" intermitente: no suporte dos bicos anteriormente descrito, foi colocado outro dispositivo que possui um interruptor automático de fluxo. Trata-se de uma haste flexível, de mola de aço, que, ao tocar o tronco, aciona o gatilho que abre a passagem para o fluxo da calda. Após passar pelo tronco, o dispositivo volta à posição original, fechando a pulverização. $\mathrm{Na}$ extremidade dessa estrutura, foi instalado um bico "off-center" OC-08 (Spraying Systems), visando a atingir somente o tronco do cafeeiro. O pulverizador foi regulado para aplicar $0,000084 \mathrm{~kg} \mathrm{~m}^{-2}$ do mesmo produto químico, a $0,70 \%$, à velocidade de $0,97 \mathrm{~m} \mathrm{~s}^{-1}$.

5) Aplicação de granulado triadimenol mais disulfoton (ANDREI, 2005): o aplicador de granulado foi montado sobre o mesmo microtrator. A máquina abre um sulco no solo, aplica o granulado sobre a copa do cafeeiro e incorpora o produto (MATUO, 2001).

O delineamento experimental utilizado foi o de blocos ao acaso, com nove tratamentos (Tabela 1) e cinco repetições. Cada parcela foi constituída de dez plantas cada, sendo consideradas úteis as três plantas centrais. As avaliações foram efetuadas contando-se o número de ninfas vivas de cigarras nas trincheiras abertas ao lado da planta, aos 68; 105 e 139 dias após a aplicação (DAA).

O segundo ensaio de campo também foi conduzido em 2004, no mesmo local, em cafezal 'Mundo Novo', com 29 anos de idade, plantado em espaçamento de 3,70 x 2,50 m e empregadas as seguintes formas de aplicação:

1) Aplicação em forma de "drench" contínuo, conforme descrita no primeiro ensaio. O pulverizador foi regulado para aplicar $0,00010 \mathrm{~kg} \mathrm{~m}^{-2}$ de Verdadero $600 \mathrm{WG}$ (thiamethoxam mais cyproconazole) à velocidade de $0,97 \mathrm{~m} \mathrm{~s}^{-1}$. A ponta foi deslocada sob a "saia", de modo que o jato alcançasse o tronco das plantas.

2) Aplicação em faixa: igual à descrita no ensaio anterior. Foi aplicado thiamethoxam mais cyproconazole a $0,3 \mathrm{~m}$ do tronco, na dosagem de $0,00010 \mathrm{~kg} \mathrm{~m}^{-2}$.

3) Aplicação em filete: efetuada com a mesma ponta e suporte descritos no ensaio de 2003. Foi aplicado thiamethoxam mais cyproconazole na dosagem de $0,00010 \mathrm{~kg} \mathrm{~m}^{-2}$.

4) Aplicação "drench intermitente": foi utilizado pulverizador costal manual, contendo limitador no curso da sua alavanca de acionamento que, por sua vez, proporcionava que a mesma quantidade de produto fosse aplicada em cada planta de café, aplicando a dosagem de $0,00010 \mathrm{~kg} \mathrm{~m}^{-2}$.

5) Aplicação "drench intermitente": igual à descrita no ensaio anterior, aplicando cyproconazole, na dosagem de $0,0008 \mathrm{~kg}$ de i.a. $\mathrm{m}^{-2}$. 
6) Foi utilizado um microtrator cafeeiro aplicando granulado (mistura triadimenol e disulfoton) na projeção da copa, na dosagem de $0,6+0,0003 \mathrm{~kg}$ de i.a. $\mathrm{m}^{-2}$ (padrão).

7) Foi utilizado um microtrator cafeeiro aplicando granulado (mistura imidacloprida e triadimenol) na projeção da copa, nas dosagens de $0,00010+0,00010 \mathrm{~kg} \mathrm{~m}^{-2}$, respectivamente.

8) Utilizou-se um microtrator cafeeiro aplicando granulado (mistura de thiamethoxam + cyproconazole), nas dosagens de $0,3+0,0003 \mathrm{~kg}$ de i.a. $\mathrm{m}^{-2}$. plantas.

9) Testemunha (sem inseticida). Todas as aplicações foram feitas nos dois lados da fileira de

O delineamento experimental foi o de blocos ao acaso, com nove tratamentos e cinco repetições. Cada parcela foi constituída de 30 plantas, sendo consideradas úteis as seis plantas centrais da linha central, tendo as demais plantas e linhas laterais como bordadura.

Foram avaliadas algumas características operacionais do protótipo. Dentre as características de desempenho, foram avaliados a velocidade de aplicação e o tempo de manobra, utilizando-se de trena e de cronômetro.

Para o pulverizador costal manual, responsável pela aplicação "drench" intermitente e o protótipo, a avaliação foi feita num cafezal da FCAV/UNESP- Jaboticabal, onde foram mensurados a velocidade de aplicação, o tempo de manobra e o tempo de reabastecimento do tanque.

A título de comparação, foram obtidos os mesmos índices para um equipamento tratorizado, levantando essas informações de desempenho junto aos usuários.

De posse dos dados sobre a velocidade e o tempo de manobra dos três equipamentos, foram estimadas as capacidades de campo operacionais, levando-se em conta os seguintes parâmetros: espaçamento do cafezal $=3 \mathrm{~m}$ entre linhas; comprimento das ruas $=100 \mathrm{~m}$; volume de aplicação do pulverizador costal manual $=0,0334 \mathrm{~L} \mathrm{~m}^{-2}$; volume de aplicação do protótipo $=0,0158 \mathrm{~L} \mathrm{~m}^{-2}$; volume de aplicação do tratorizado $0,0160 \mathrm{~L} \mathrm{~m}^{-2}$; capacidade dos depósitos: pulverizador costal manual $=20 \mathrm{~L}$; protótipo $=40 \mathrm{~L}$; tratorizado $=400 \mathrm{~L}$; tempo de preparo $=1.800$ segundos para todos os equipamentos; jornada de trabalho $=28.800$ segundos; tempo para reabastecimento $=$ 150 segundos para pulverizador costal manual, 120 segundos para protótipo e 15.900 segundos para o tratorizado.

Para o cálculo da capacidade de campo operacional, foi utilizada a eq.(1), de MATUO (1990):

$$
\mathrm{t}=\frac{10000}{\mathrm{Va} \mathrm{L}}+\frac{10000 \mathrm{TV}}{\mathrm{C} \mathrm{L}}+\frac{\mathrm{d} \mathrm{V}}{\mathrm{Vd} \mathrm{Ca}}+\frac{\mathrm{Tr} \mathrm{V}}{\mathrm{Ca}}
$$

em que,

$\mathrm{t}$ - tempo para aplicar 1 ha, $\min \mathrm{ha}^{-1}$

Va - velocidade de aplicação, $\mathrm{m} \mathrm{min}^{-1}$;

L - largura da faixa de aplicação, m;

Tv - tempo de manobra, min;

$\mathrm{C}$ - comprimento da faixa de tratamento, $\mathrm{m}$;

d - distância total percorrida para cada reabastecimento, m;

$\mathrm{V}$ - volume de aplicação, $\mathrm{L} \mathrm{ha}^{-1}$;

$\mathrm{Vd}$ - velocidade de deslocamento para reabastecimento, $\mathrm{m} \mathrm{min}^{-1}$;

$\mathrm{Ca}$ - capacidade de tanque, $\mathrm{L}$, e

$\mathrm{Tr}$ - tempo de reabastecimento do tanque, min.

$\mathrm{Na}$ eq.(1), a fração $(\mathrm{d} \mathrm{V}) /(\mathrm{Vd} \mathrm{Ca})$, que corresponde ao tempo para percorrer até o ponto de abastecimento, foi considerada nula supondo que o abastecimento se efetuaria no carreador.

De posse do tempo necessário para tratar 1 ha, foi calculada a capacidade operacional, seguindo a eq.(2): 


$$
\mathrm{CcO}=\frac{7,5}{8} \frac{60}{\mathrm{t}}
$$

em que,

$\mathrm{CcO}$ - capacidade de campo operacional, ha $\mathrm{h}^{-1}$;

$\mathrm{t}$ - tempo calculado através da eq.(1), $\min _{\mathrm{ha}}{ }^{-1}$;

8 - jornada diária de trabalho, h, e

7,5 - jornada de trabalho menos o tempo de preparo, h.

\section{RESULTADOS E DISCUSSÃO}

As diferenças no número médio de ninfas vivas de $Q$. gigas tornaram-se mais evidentes a partir dos 105 DAA; quando os tratamentos contendo thiamethoxam diferiram significativamente da testemunha. Aos 139 DAA, além dos tratamentos com thiamethoxam, o imidacloprid aplicado em filete e em "drench" contínuo, e a mistura imidacloprid com triadimenol aplicado na formulação granulada também diferiram da testemunha. A mistura triadimenol com disulfoton, na formulação granulada, utilizada como padrão, não diferiu de nenhum dos tratamentos nas três avaliações desse ensaio.

TABELA 1. Número médio de ninfas de Quesada gigas e eficiência de controle (\%) nos respectivos tratamentos, aos 68; 105 e 139 dias após a aplicação (DAA). Number of Quesada gigas and control efficiency at 68; 105 and 139 days after application (DAA).

\begin{tabular}{|c|c|c|c|c|c|c|c|}
\hline \multirow{3}{*}{ Tratamentos } & \multirow{3}{*}{ Produtos $^{1}$} & \multicolumn{6}{|c|}{ Número de ninfas ${ }^{2}$} \\
\hline & & \multicolumn{2}{|c|}{$68 \mathrm{DAA}$} & \multicolumn{2}{|c|}{$105 \mathrm{DAA}$} & \multicolumn{2}{|c|}{139 DAA } \\
\hline & & $\mathrm{N}^{\mathrm{o}}$ & $\% \mathrm{E}$ & $\mathrm{N}^{\mathrm{o}}$ & $\% \mathrm{E}$ & $\mathrm{N}^{\mathrm{o}}$ & $\% \mathrm{E}$ \\
\hline 1. Filete & imidacloprida $^{1.1}$ & $2,18 a b^{3}$ & $40^{4}$ & $1,31 \mathrm{a}$ & 86 & $1,99 \mathrm{~b}$ & 76 \\
\hline 2. Faixa & imidacloprida & $2,59 \mathrm{ab}$ & 40 & $1,99 \mathrm{ab}$ & 68 & $2,05 \mathrm{ab}$ & 82 \\
\hline 3. "Drench" contínuo & imidacloprida & $2,26 \mathrm{ab}$ & 59 & $1,83 \mathrm{ab}$ & 72 & $1,93 \mathrm{~b}$ & 82 \\
\hline 4. "Drench"intermitente & imidacloprida & $2,61 \mathrm{ab}$ & 37 & $2,57 \mathrm{ab}$ & 56 & $2,38 \mathrm{ab}$ & 69 \\
\hline 5. Granulado & $\begin{array}{l}\text { triadimenol + } \\
\text { disulfoton }^{1.2}\end{array}$ & $2,01 \mathrm{ab}$ & 40 & $2,71 \mathrm{ab}$ & 58 & $2,42 \mathrm{ab}$ & 76 \\
\hline 6. Drench contínuo & thiamethoxam ${ }^{1.3}$ & $0,95 \mathrm{ab}$ & 88 & $1,05 \mathrm{~b}$ & 93 & $1,29 \mathrm{~b}$ & 87 \\
\hline 7. Granulado & $\begin{array}{l}\text { imidacloprida } 3+ \\
\text { triadimenol } 3^{1.4}\end{array}$ & $2,60 \mathrm{ab}$ & 43 & $1,39 a b$ & 86 & $1,56 \mathrm{~b}$ & 86 \\
\hline 8. Granulado & $\begin{array}{l}\text { thiamethoxam }+ \\
\text { cyproconazole }^{1.5}\end{array}$ & $2,04 \mathrm{ab}$ & 65 & $1,22 \mathrm{~b}$ & 89 & $1,55 \mathrm{~b}$ & 89 \\
\hline 9. Testemunha & --- & $3,16 \mathrm{a}$ & - & $3,26 \mathrm{a}$ & - & $3,65 \mathrm{a}$ & - \\
\hline C.V. $(\%)$ & & 42 & & 48 & & 37 & \\
\hline DMS & & 2,01 & & 1,97 & & 1,65 & \\
\hline
\end{tabular}

${ }^{1.1}$ Premier GRDA $\left(0,00012 \mathrm{~kg} \mathrm{~m}^{-2}\right) ;{ }^{1.2}$ Baysiston GR $\left(0,0040 \mathrm{~kg} \mathrm{~m}^{-2}\right) ;{ }^{1.3}$ Actara GRDA $\left(0,00012 \mathrm{~kg} \mathrm{~m}^{-2}\right) ;{ }^{1.4}$ Premier 3 mais Bayfidan $3\left(0,0040 \mathrm{~kg} \mathrm{~m}^{-2}\right) ;{ }^{1.5}$ Verdadero GR $\left(0,0030 \mathrm{~kg} \mathrm{~m}^{-2}\right) ;{ }^{2}$ Números transformados em $\log (\mathrm{x}+1){ }^{3}$ Médias, na mesma coluna, seguidas de pelo menos uma letra igual, não se diferem estatisticamente, pelo teste de Tukey, a $5 \%$ de probabilidade; ${ }^{4}$ Eficiência de controle calculada pela fórmula de Abbott.

Com relação à tecnologia de aplicação, a líquida mostrou-se mais simples e prática pela facilidade do preparo da calda e na aplicação propriamente dita, além de menor interferência na lavoura por deixar a superfície do solo intacta. Dentre as diferentes técnicas de aplicação líquida, as aplicações sob forma de filete e "drench" contínuo mostraram-se eficientes, de fácil execução e promissoras para o controle de cigarras nessa cultura.

A aplicação sob forma de "drench" intermitente, embora tenha sido satisfatória, mostrou -se menos conveniente, pois o mecanismo de liberação da calda, para que seja acionado, tem de entrar em contato com a planta. Isso pode constituir-se em um fator complicador, pois caso a mola não entre em contato com o tronco da planta, não será acionado o gatilho da lança de aplicação. MARTINELLI et al. (2001) verificaram que o imidacloprid aplicado na forma de "drench" pode ser 
recomendado para o controle de cigarras do cafeeiro com a dosagem de 0,00008 $\mathrm{kg}$ de i.a. $\mathrm{m}^{-2}$. MATUO et al. (2002) constataram que, dentre as diferentes formas de aplicação de líquidos, a aplicação pelo processo "drench" mostrou-se mais eficiente no controle das cigarras do cafeeiro em todo o período de condução do experimento.

Diante dos resultados do ensaio de campo I, decidiu-se pela interrupção do desenvolvimento do processo "drench" intermitente para o protótipo. O tratamento foi mantido no presente experimento por meio do processo manual de um pulverizador costal próprio, uma vez que esse processo de aplicação já se tornou usual nos experimentos com a aplicação líquida (SOUZA \& REIS, 2002; SOUZA \& REIS, 2003).

Aos 140 dias após a aplicação, os tratamentos com thiamethoxam mais cyproconazole aplicados em filete e faixa apresentaram diferenças estatísticas em relação à testemunha (Tabela 2). Aos 174 dias, os tratamentos aplicados em "drench" contínuo, filete e "drench" intermitente com thiamethoxam mais cyproconazole diferiram significativamente da testemunha. Já aos 202 dias, os tratamentos com essa mistura, aplicada em faixa, filete e na formulação granulada, diferiram estatisticamente da testemunha.

TABELA 2. Número médio de ninfas de Quesada gigas e eficiência dos produtos para o controle nos respectivos tratamentos aos 140; 174 e 202 dias após a aplicação (DAA). Number of Quesada gigas and control efficiency at 140; 174 and 202 days after application (DAA).

\begin{tabular}{|c|c|c|c|c|c|c|c|}
\hline \multirow{3}{*}{ Tratamentos } & \multirow{3}{*}{ Produtos $^{1}$} & \multicolumn{6}{|c|}{ Número de ninfas ${ }^{6}$} \\
\hline & & \multicolumn{2}{|c|}{140 DAA } & \multicolumn{2}{|c|}{174 DAA } & \multicolumn{2}{|c|}{202 DAA } \\
\hline & & № & $\% \mathrm{E}$ & № & $\% \mathrm{E}$ & Noㅇ & $\% \mathrm{E}$ \\
\hline 1. "Drench"contínuo & $\begin{array}{l}\text { thiamethoxam }+ \\
\text { cyproconazole }^{1}\end{array}$ & $1,41 a b^{7}$ & $50,7^{8}$ & $0,86 \mathrm{bc}$ & 69,2 & $0,91 \mathrm{ab}$ & 65,5 \\
\hline 2. Faixa & $\begin{array}{c}\text { thiamethoxam + } \\
\text { cyproconazole }\end{array}$ & $0,36 \mathrm{~b}$ & 87,4 & $1,50 \mathrm{abc}$ & 46,2 & $0,70 \mathrm{~b}$ & 73,4 \\
\hline 3. Filete & $\begin{array}{c}\text { thiamethoxam + } \\
\text { cyproconazole }\end{array}$ & $0,55 \mathrm{~b}$ & 80,7 & $0,64 \mathrm{c}$ & 77,1 & $0,55 \mathrm{~b}$ & 79,2 \\
\hline 4. "Drench" intermitente & $\begin{array}{l}\text { thiamethoxam + } \\
\text { cyproconazole }\end{array}$ & $0,98 \mathrm{ab}$ & 65,7 & $0,74 \mathrm{c}$ & 73,4 & $1,47 \mathrm{ab}$ & 44,3 \\
\hline 5. "Drench" intermitente & thiamethoxam $^{2}$ & $1,41 \mathrm{ab}$ & 60,1 & $1,15 \mathrm{abc}$ & 58,8 & $1,26 \mathrm{ab}$ & 52,3 \\
\hline 6. "Drench" intermitente & cyproconazole $^{3}$ & $2,71 \mathrm{a}$ & 5,2 & $2,77 \mathrm{ab}$ & 0,7 & $2,02 \mathrm{ab}$ & 23,5 \\
\hline 7. Grânulos & $\begin{array}{c}\text { triadimenol }+ \\
\text { disulfoton }^{4}\end{array}$ & $1,79 \mathrm{ab}$ & 37,4 & $1,64 \mathrm{abc}$ & 41,2 & $2,41 \mathrm{ab}$ & 8,7 \\
\hline 8. Grânulos & $\begin{array}{l}\text { thiamethoxam + } \\
\text { cyproconazole }^{5}\end{array}$ & $1,46 \mathrm{ab}$ & 48,9 & $1,24 \mathrm{abc}$ & 55,6 & $0,67 \mathrm{~b}$ & 74,6 \\
\hline 9. Testemunha & & $2,86 \mathrm{a}$ & 0,0 & $2,79 \mathrm{a}$ & 0,0 & $2,64 \mathrm{a}$ & 0,0 \\
\hline C.V. $(\%)$ & & 60 & & 62 & & 65 & \\
\hline DMS & & 1,91 & & 1,93 & & 1,92 & \\
\hline
\end{tabular}

${ }^{1}$ Verdadero $600 \mathrm{WG}\left(0,00010 \mathrm{~kg} \mathrm{~m}^{-2}\right) ;{ }^{2}$ Actara $250 \mathrm{WG}\left(0,00012 \mathrm{~kg} \mathrm{~m}^{-2}\right) ;{ }^{3}$ Alto $100 \mathrm{CS}\left(0,00030 \mathrm{~kg} \mathrm{~m}^{-2}\right) ;{ }^{4}$ Baysiston GR $\left(0,004 \mathrm{~kg} \mathrm{~m}^{-2}\right) ;{ }^{5}$ Verdadero GR $\left(0,003 \mathrm{~kg} \mathrm{~m}^{-2}\right) ;{ }^{6}$ Números transformados em $\log (\mathrm{x}+1) ;{ }^{7}$ Médias, na mesma coluna, seguidas de pelo menos uma letra igual não diferem estatisticamente, pelo teste de Tukey, a $5 \%$ de probabilidade; ${ }^{8}$ Percentagem de controle calculada pela fórmula de Abbott.

O tratamento "drench" intermitente somente com o fungicida cyproconazole, na ausência de inseticida, comportou-se de forma semelhante à testemunha, conforme o esperado.

A aplicação em filete com thiamethoxam mais cyproconazole foi o único tratamento que diferiu da testemunha em todas as avaliações. Essa forma de aplicação não encontra nenhum impedimento no momento da aplicação, pois, ao utilizar somente uma ponta com orifício relativamente grande e sem núcleo, torna-se praticamente imune ao entupimento. Além disso, o 
filete único sob pressão está mais apto a romper a eventual barreira de folhas caídas e alcançar a superfície do solo. A aplicação em "drench" contínuo e em faixa da mistura thiamethoxam e cyproconazole também são métodos interessantes, pois diferiram da testemunha em uma ou mais avaliações.

Os tratamentos com formulações em grânulos não apresentaram resultados tão consistentes quanto os líquidos, exceção ao tratamento em grânulos com thiamethoxam mais cyproconazole aos 202 dias após a aplicação, que apresentou boa eficiência de controle da cigarra.

No entanto, a aplicação de granulados é uma forma de aplicação consolidada e bastante difundida, como pode ser constatado em vários trabalhos, a exemplo de MARTINS et al. (1998), em que a análise dos resultados revelou que as aplicações dos inseticidas granulados reduziram significativamente o número de ninfas vivas em relação à testemunha. MARTINELLI e LUSVARGHI (1998) também constataram benefícios no uso dos inseticidas granulados no controle de cigarras, em que os resultados diferiram significativamente da testemunha em todas as avaliações.

AIZAWA et al. (2000) concluíram que thiamethoxam associado ao cyproconazole na dosagem de $0,0025 \mathrm{~kg} \mathrm{~m}^{-2}$ de produto comercial foi eficiente no controle de $Q$. gigas.

O protótipo desenvolvido para a aplicação de produtos fitossanitários na formulação líquida está apresentado na Figura 1.

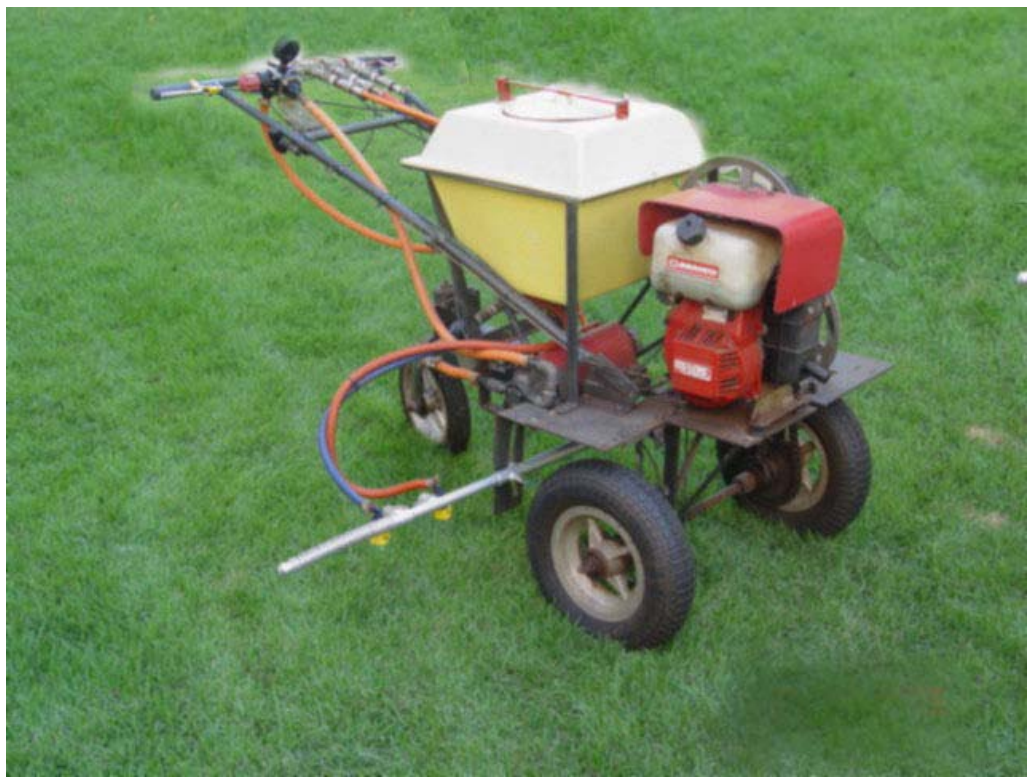

FIGURA 1. Protótipo desenvolvido para aplicação de produtos líquidos na cultura do café. Prototype developed for spraying coffee trees.

Os tratamentos "drench" intermitente com thiamethoxam mais cyproconazole e "drench" intermitente somente com thiamethoxam, aplicados no tronco, utilizando-se do pulverizador costal manual, não se mostraram eficientes no controle da cigarra $Q$. gigas.

Independentemente do produto neonicotinóide aplicado, imidacloprid ou thiamethoxam, o método de aplicação líquida por filete mostrou-se bastante promissor no controle de Q. gigas, mantendo o bom desempenho no controle desse inseto-praga nos anos de 2003 e 2004.

O protótipo apresentou desempenho dentro do esperado, permitindo fácil regulagem e calibração. Proporcionou a aplicação em faixa, filete e em "drench" com precisão e fácil operação, pois para se optar por uma das formas de aplicação, bastou selecionar o respectivo registro, conforme ilustra a Figura 2. O suporte das pontas de pulverização (Figura 3), variável na extensão e na angulação em relação ao solo, permitiu fácil posicionamento da aplicação nos pontos 
previamente determinados. Somente o dispositivo construído para a aplicação intermitente (Figura 4) mostrou ser um tanto frágil para a finalidade; entretanto, os resultados dos ensaios indicaram que o seu desenvolvimento não se faria necessário, visto que os resultados obtidos com a aplicação intermitente, em alguns casos, não diferem significativamente daqueles registrados nas parcelas não-tratadas (testemunha) e, quando diferem, os benefícios advindos da adoção dessa técnica no controle das cigarras são pouco expressivos.

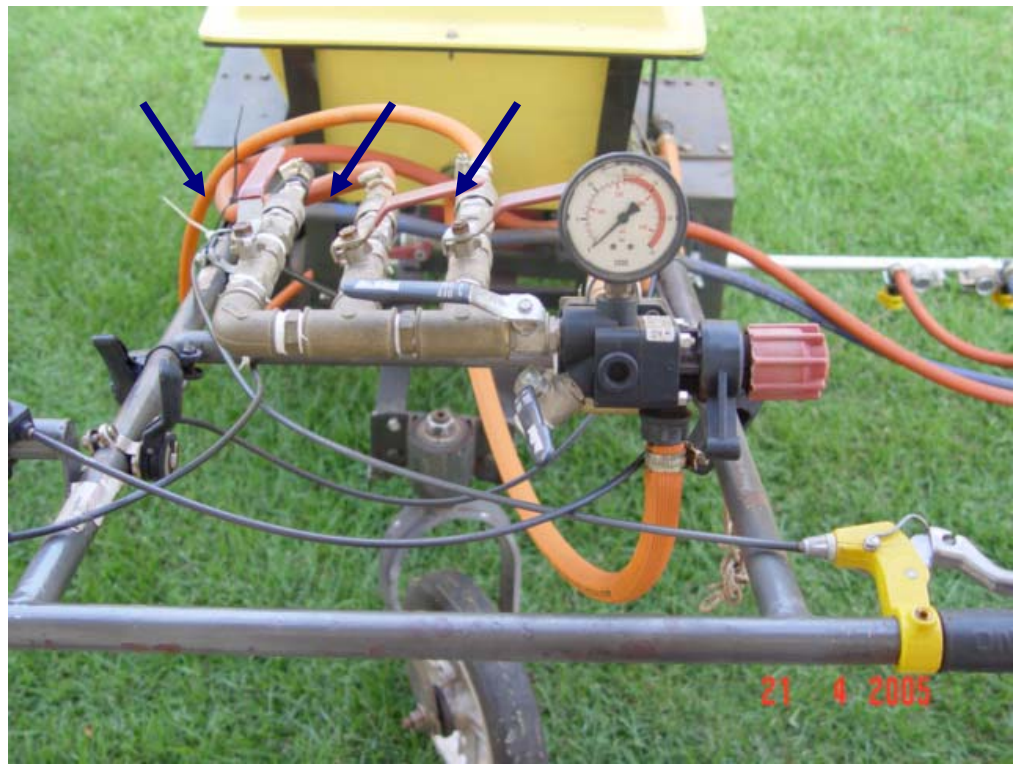

FIGURA 2. Detalhe dos comandos do sistema de pulverização com os registros para cada tipo de aplicação. Details of the control system sprayer showing the shut of valves for each type of application.

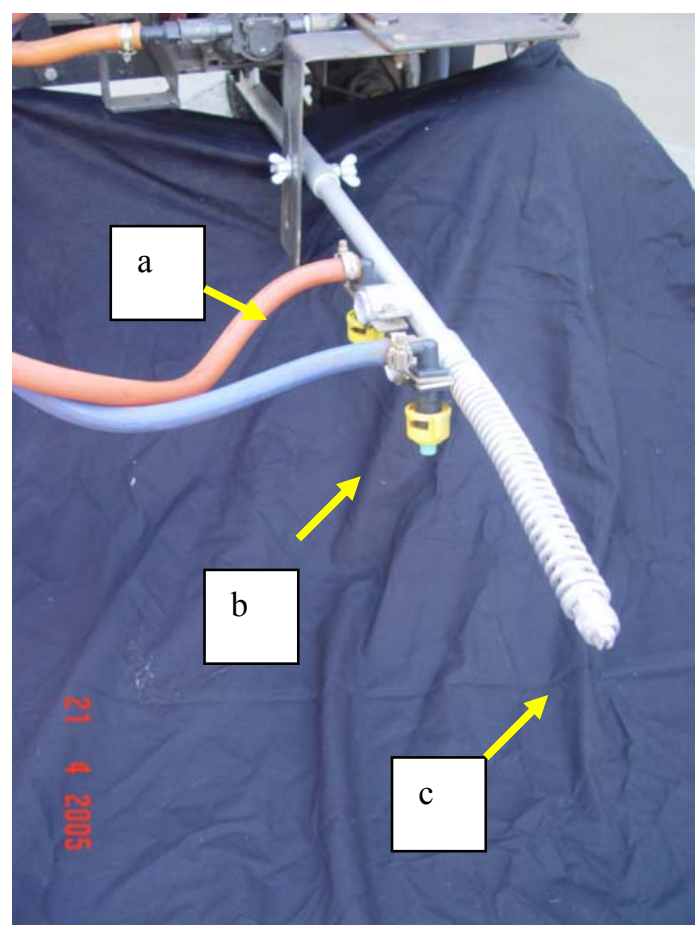

FIGURA 3. Suporte ajustável dos bicos de pulverização mostrando as três modalidades de aplicação: a) faixa; b) filete, e c) "drench". Adjustable nozzle holder for each type of application: a) band, b) solid stream, c) drench.

Quanto à condução, o protótipo apresentou manobrabilidade bastante satisfatória, permitindo facilidade de condução no meio do cafezal e manobras rápidas. A adoção da terceira roda direcional deu agilidade nas manobras (Figura 5). A faixa de velocidade disponível, que pode ser variada 
continuamente acima ou abaixo de $0,0010 \mathrm{~m} \mathrm{~s}^{-1}$, com a variação da rotação do motor, foi suficientemente confortável para conduzir e acompanhar a máquina dentro e fora da lavoura. Os comandos colocados ao alcance das mãos permitiram boa ergonomia.

Os resultados das características de desempenho operacional expressas em função das velocidades e dos tempos de manobra dos equipamentos são apresentados na Tabela 3. Constatouse que o tempo de manobra do pulverizador costal manual e do protótipo são os mesmos. Os dois equipamentos conseguiram efetuar a operação de manobra em apenas 5 segundos.

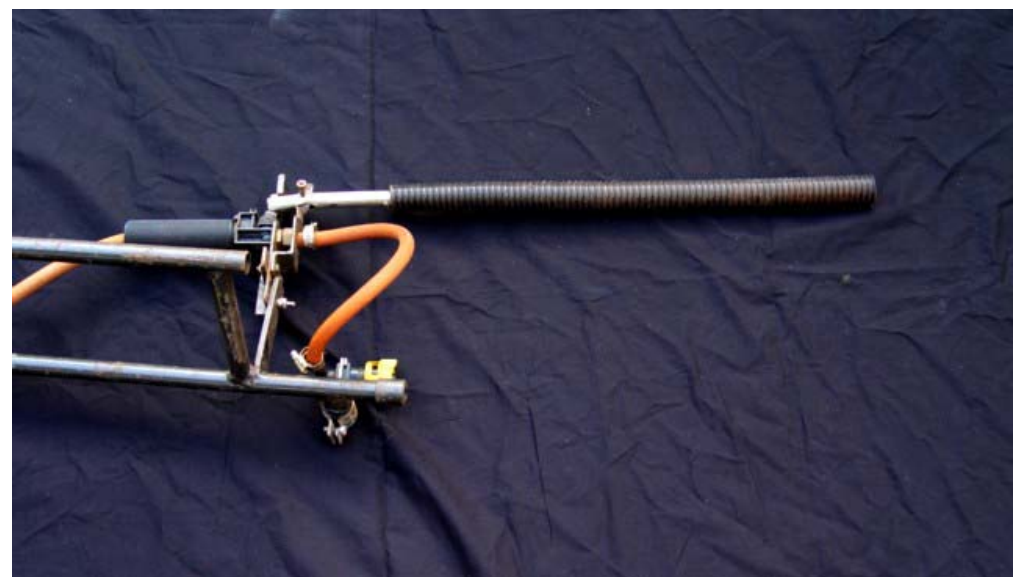

FIGURA 4. Dispositivo para aplicação "drench" intermitente. Device for intermitent drench application.

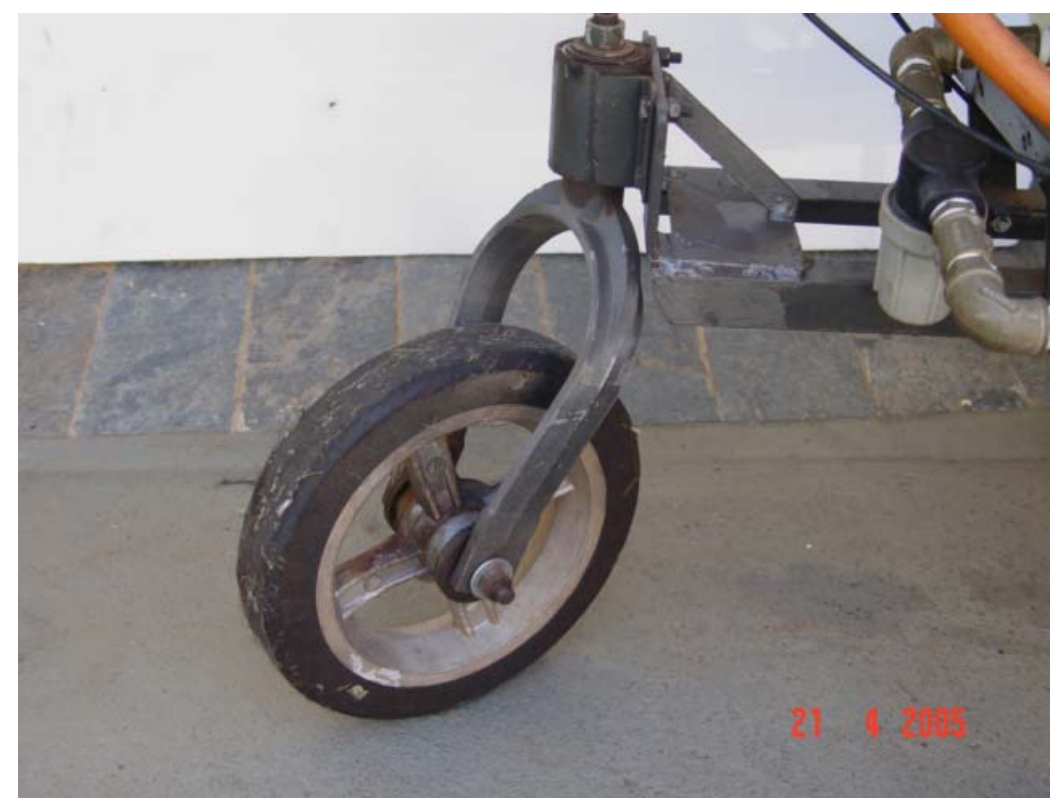

FIGURA 5. Terceira roda direcional. Third directional wheel.

A velocidade de aplicação do protótipo é praticamente quatro vezes maior que a do pulverizador costal manual.

As estimativas das capacidades de campo operacionais, calculadas a partir das condições relacionadas anteriormente e dos dados de velocidade e de tempo de manobra observados para cada um dos equipamentos, indicam que o tratorizado é o que apresenta maior capacidade operacional, em decorrência da maior velocidade observada.

Pela Tabela 3, nota-se que o equipamento tratorizado apresentou a maior capacidade de campo operacional entre todos os equipamentos. $O$ equipamento costal manual apresenta capacidade de campo operacional inferior àquela dos três equipamentos devido à menor velocidade 
de trabalho em relação aos demais equipamentos. Comparado ao protótipo realizando a aplicação em apenas um dos lados da linha de plantio, constata-se que o tratorizado apresenta o dobro da capacidade de campo operacional. Porém, nada impede que o protótipo seja munido de outro suporte que possibilite a aplicação nos dois lados da rua de café, pois todo o sistema de pulverização é perfeitamente dimensionado para tal tarefa. Dessa forma, a capacidade de campo operacional do protótipo, aplicando nos dois lados da linha de plantio, fica bem próxima do equipamento tratorizado.

TABELA 3. Velocidade de aplicação, tempo de manobra e capacidade de campo operacional estimada dos equipamentos em teste, na cultura do café. Speed application, turn over turn, overall work rate estimated for the test equipment in the coffee plantations.

\begin{tabular}{lccc}
\hline \multicolumn{1}{c}{ Equipamentos } & $\begin{array}{c}\text { Velocidade } \\
\left(\mathrm{m} \mathrm{s}^{-1}\right)\end{array}$ & $\begin{array}{c}\text { Tempo de Manobra } \\
(\mathrm{s})\end{array}$ & $\begin{array}{c}\text { CcO Estimada } \\
\left(\mathrm{ha} \mathrm{h}^{-1}\right)^{1}\end{array}$ \\
\hline Tratorizado & 4,5 & 30 & 0,84 \\
Costal manual & 0,93 & 5 & 0,12 \\
Protótipo (aplicando 1 lado) & 3,6 & 5 & 0,42 \\
Protótipo (aplicando 2 lados) & 3,6 & 5 & 0,76 \\
\hline
\end{tabular}

${ }^{1}$ Capacidade de campo operacional estimada com os seguintes valores: espaçamento do cafezal $=3 \mathrm{~m}$ entre linhas; comprimento das ruas $=100 \mathrm{~m}$; volume de aplicação do pulverizador costal manual $=334 \mathrm{~L} 10.000 \mathrm{~m}^{-2}$; volume de aplicação do protótipo $=158 \mathrm{~L} 10.000 \mathrm{~m}^{-2}$; volume de aplicação do tratorizado $=160 \mathrm{~L} 10.000 \mathrm{~m}^{-2}$; capacidade dos depósitos: pulverizador costal manual $=20 \mathrm{~L}$; protótipo $=40 \mathrm{~L}$; tratorizado $=400 \mathrm{~L}$; tempo de preparo $=$ 1.800 segundos para todos os equipamentos; jornada de trabalho $=28.800$ segundos; tempo para reabastecimento $=$ 120 segundos e 30 segundos para pulverizador costal manual, 240 segundos para protótipo e 900 segundos para o tratorizado.

\section{CONCLUSÕES}

O protótipo aplicador de produtos fitossanitários na forma líquida atendeu aos objetivos propostos, visto que conseguiu realizar o controle químico das cigarras do cafeeiro com a mesma eficácia dos produtos granulados, que são considerados padrões de controle.

O protótipo, se equipado para aplicar nos dois lados da planta, apresentará capacidade de campo operacional semelhante à do equipamento tratorizado.

Os métodos de aplicação de líquidos, com destaque para a aplicação em "drench" contínuo, proporcionam níveis satisfatórios de controle de cigarras.

As aplicações líquidas apresentaram controle satisfatório tanto quanto a aplicação de grânulos, com vantagens como maior segurança por utilizar produtos menos tóxicos, máquinas mais simples para aplicação e por dispensar a incorporação ao solo do produto químico.

\section{REFERÊNCIAS}

AIZAWA, J.S.; ARAMAKI, P.H.; BOSQUE, J.C.; GUICHERIT, E. Efeito do inseticida thiamethoxam, aplicado em diferentes doses e formulações, no controle da cigarra do cafeeiro Quesada gigas (Oliv., 1970) (Homoptera - Cicadidae). In: CONGRESSO BRASILEIRO DE PESQUISAS CAFEEIRAS, 26., 2000, Marília. Anais... Rio de Janeiro: MAPA/PROCAFÉ, 2000. p.182-3.

ANDREI, E. Compêndio de defensivos agrícolas. 7.ed. São Paulo: Andrei, 2005. 1.141 p.

MACHADO NETO, J.G.; MATUO, T.; MATUO, Y.K. Semiquantitative evaluation of dermal exposure to granulated insecticides in coffee (Coffea arabica L.) crop and efficiency of individual protective equipment. Bulletin Environmental Contamination Toxicology, New York, v.57, n.6, p.946-51, 1996. 
MARTINELLI, N.M.; LIMA, M.F.D.; MATUO, T.K. Avaliação da eficiência do imidacloprid, aplicado via líquida, para o controle de cigarras do cafeeiro. In: CONGRESSO BRASILEIRO DE PESQUISAS CAFEEIRAS, 27., 2001, Uberaba. Anais... Rio de Janeiro: MAPA/PROCAFÉ, 2001. p.125-6.

MARTINELLI, N.M.; LUSVARGHI, H.N. Controle de cigarras do cafeeiro com terbufós em duas formulações. In: CONGRESSO BRASILEIRO DE PESQUISAS CAFEEIRAS, 24., 1998, Poços de Caldas. Anais... Rio de Janeiro: MAPA/PROCAFÉ, 1998. p.115.

MARTINS, D.R.; MARTINELLI, N.M.; MATUO, T. Métodos de aplicação de inseticidas granulados sistêmicos para o controle de cigarras do cafeeiro. In: CONGRESSO BRASILEIRO DE PESQUISAS CAFEEIRAS, 24., 1998, Poços de Caldas. Anais... Rio de Janeiro: MAPA/PROCAFÉ, 1998. p.114-5.

MATUO, T. Técnicas de aplicação de defensivos agrícolas. Jaboticabal: Funep, 1990. 139 p.

MATUO, T.K. Desenvolvimento de um aplicador motorizado para formulações granuladas de agrotóxicos na cultura do café. 2001. 48 f. Dissertação (Mestrado em Proteção de Plantas) Faculdade de Ciências Agronômicas, Universidade Estadual Paulista, Botucatu, 2001.

MATUO, T.K.; MARTINELLI, N.M.; LIMA, M.F.D.; RAETANO, C.G. Eficiência do imidacloprid aplicado via líquida pelo microtrator cafeeiro para o controle de cigarras e do bicho mineiro. In: CONGRESSO BRASILEIRO DE PESQUISAS CAFEEIRAS, 28., 2002, Caxambu. Anais... Rio de Janeiro: MAPA/PROCAFÉ, 2002. p.268-71.

SOUZA, J.C.; REIS, P.R. Eficiência de thiamethoxam $250 \mathrm{WG}$ aplicado em diferentes modalidades no controle do bicho mineiro do cafeeiro. In: CONGRESSO BRASILEIRO DE PESQUISAS CAFEEIRAS, 28., 2002, Caxambu. Anais... Rio de Janeiro: MAPA/PROCAFÉ, 2002. p.343-5.

SOUZA, J.C.; REIS, P.R. Controle da cigarra Quesada gigas (Olivier) (Hemiptera; Homoptera: Cicadidae) com formulações e modos de aplicação de thiamethoxam e da mistura de thiamethoxam e cyproconazole. In: CONGRESSO BRASILEIRO DE PESQUISAS CAFEEIRAS, 29., 2003, Araxá. Anais... Rio de Janeiro: MAPA/PROCAFÉ, 2003. p.172-3. 